\title{
A Novel Function for Programmed Death Ligand-1
}

\section{Regulation of Angiogenesis}

\author{
Yiping Jin, ${ }^{*}$ Sunil K. Chauhan, ${ }^{*}$ Jaafar El Annan, ${ }^{*}$ \\ Peter T. Sage, ${ }^{\dagger}$ Arlene H. Sharpe, ${ }^{\dagger}$ \\ and Reza Dana* \\ From the Department of Ophthalmology,* Schepens Eye Research \\ Institute and Massachusetts Eye and Ear Infirmary, and the \\ Department of Pathology, ${ }^{\dagger}$ Harvard Medical School, Boston, \\ Massachusetts
}

Programmed death ligand-1 (PD-L1) plays a critical role in T-cell regulatory function. Here, we report a newly discovered effect of PD-L1 on angiogenesis. We demonstrate that PD-L1 and its receptor CD80, but not PD-1, are expressed by primary murine lung and heart vascular endothelial cells and the miscrovascular endothelial cell line (MS1) at both the mRNA and protein levels in vitro. The inhibition of PD-L1 or CD80 expression in MS1 cells, by small-interfering RNA transfection, led to a significant up-regulation of vascular endothelial growth factor receptor 2 expression and cell proliferation levels in MS1 cells. Furthermore, MS1 cells were found to have a significantly lower proliferation and vascular endothelial growth factor receptor 2 expression levels when they were co-cultured with PD-L1-expressing normal corneal epithelial cells, as compared to MS1 cells co-cultured with PD-L1 ${ }^{-/-}$corneal epithelial cells. In a sutureinduced corneal angiogenesis model, we observed a significantly higher level of angiogenic response in PD-L1 $^{-/-}$knockout mice as compared to wild-type mice, although there was no significant difference in the expression of inflammatory cytokines (interleukin-1 $\alpha$, interleukin-1 $\beta$, or tumor necrosis factor- $\alpha$ ) or the infiltration of innate immune cells (neutrophils and macrophages) between the two groups. We conclude that the expression of PD-L1 in both vascular endothelial cells and corneal epithelial cells regulates corneal angiogenesis. (Am J Pathol 2011, 178:1922-1929; DOI: 10.1016/j.ajpath.2010.12.027)

Anti-inflammatory and anti-angiogenic properties of the cornea are crucial to maintain its immune privilege status. The cornea possesses an extensive array of mechanisms by which immune effectors and neovascularization are regu- lated, or even silenced. These mechanisms include an intact corneal epithelial layer; soluble immunomodulatory factors in the aqueous humor, the blood-ocular barrier; epithelial expression of membrane and soluble vascular endothelial growth factor receptors (VEGFRs) ${ }^{1,2}$; and the high expression of Fas ligand and thrombospondin-1. ${ }^{3-6}$

Recently, another immunoregulatory molecule, programmed death ligand-1 (PD-L1, also known as CD274 or $\mathrm{B} 7-\mathrm{H} 1)$, has been shown to be expressed at high levels by corneal tissue by our laboratory and others. ${ }^{7,8}$ PD-L1 is a member of the B7-CD28 family, which plays important roles in regulating $\mathrm{T}$-cell activation and tolerance through its receptors PD-1 and CD80. ${ }^{9,10} \mathrm{PD}-\mathrm{L} 1$ is expressed constitutively on both hematopoietic cells (resting T cells, B cells, dendritic cell, macrophages, and regulatory $T$ cells) and nonhematopoietic cells (parenchymal and endothelial cells) and is up-regulated to higher levels by inflammatory stimuli. ${ }^{11-14}$ Peripheral tissue-specific PD-L1 expression indicates that it may have a key role in regulating or terminating immune responses in inflamed tissues. Previous studies from our laboratory and other groups have shown PD-L1 to play a critical function in peripheral immune tolerance as a negative regulator of T-cell responses. ${ }^{7,8}$

Studies have shown that several immunoregulatory factors, such as Fas ligand and thrombospondin-1, also possess a regulatory function on neovascularization through their expression by vascular endothelial cells (VECs). ${ }^{15,16}$ Interestingly, PD-L1 also has been reported to be present on VECs under basal conditions, and the surface expression of PD-L1 on VECs can be increased by interferon- $\alpha,-\beta$, and $-\gamma{ }^{17,18}$ However, the anti-angiogenic effect of PD-L1 has not been demonstrated to date. Here, we provide evidence for a novel regulatory function for PD-L1 in VEC proliferation in vitro and corneal angiogenesis in vivo.

Supported by National Institutes of Health grants R01 EY 12963 (R.D.), R01 Al 40614 (A.H.S.), and T32 Al 070085-03 (P.T.S.).

Accepted for publication December 17, 2010.

Address reprint requests to Dr. Reza Dana, Schepens Eye Research Institute, Harvard Medical School, 20 Staniford St., Boston, MA 02114. E-mail: reza.dana@schepens.harvard.edu. 


\section{Materials and Methods}

\section{Animals}

Male C57BL/6 mice ages 6 to 8 weeks were obtained from Taconic Farms (Germantown, NY). A similar age range of PD-L $1^{-1-}$ C57BL/6 mice were generated as previously described. ${ }^{19}$ All experimental protocols were approved by the Schepens Eye Research Institute and/or Harvard Medical School Animal Care and Use Committees, and all animals were treated according to the Association for Research in Vision and Ophthalmology Statement for the Use of Animals in Ophthalmic and Vision Research.

\section{Suture-Induced Inflammatory Corneal Angiogenesis}

Three interrupted sutures (11-0 nylon, Sharpoint; Vanguard, Houston, TX) were placed intrastromally with two stromal incursions extending over $120^{\circ}$ of the corneal circumference each to induce inflammatory corneal neovascularization (NV), as described previously. ${ }^{20}$ The corneas were later examined by slit-lamp biomicroscopy for corneal NV development. NV was graded between 0 and 3 , with increments of 0.5 , using a grid system per each corneal quadrant based on the centripetal extent of the neovascular branches from the limbus. Scores for each quadrant then were summed to derive the NV index (range, 0-12) for each eye, as previously described. ${ }^{20}$

\section{RNA Isolation and Real-Time PCR}

Corneas were carefully dissected to ensure that the conjunctiva and iris tissues were not included. For mRNA extraction from whole-thickness corneas, two corneas were pooled as a sample in each group. To extract mRNA from cells in plates, $500 \mu \mathrm{L}$ TRIzol (Invitrogen, Carlsbad, CA) was used to lyses cells.

Our combined method for total RNA isolation consisted of TRIzol and RNeasy MinElute Spin Columns (Qiagen, Valencia, CA), as described previously. ${ }^{21}$ Reverse transcription of total RNA was conducted using oligo(deoxythymidine) $)_{20}$ primer and Superscript III Reverse Transcriptase (Invitrogen). A total of $1 \mu \mathrm{L}$ of total complementary DNA, synthesized from $400 \mathrm{ng}$ total RNA with random hexamers using Superscript III Reverse Transcriptase (Invitrogen), was loaded in each well and assays were performed in triplicate. Quantitative PCR was performed with TaqMan Universal PCR Mastermix and FAM-MGB dye-labeled predesigned primers (Applied Biosystems, Foster City, CA) for PD-L1 (Mm01208505_m1), CD80 (Mmoo711660_m1), PD1 (Mm01285677_g1), IL-1 $\alpha$ (Mm99999060_ml), tumor necrosis factor $\alpha$ (TNF- $\alpha) \quad($ Mm99999068_ml), IL-1 $\beta$ (Mm00434228_ml), VEGFR2 (Mm00440099_ml), and VEGFA (Mm00437304_ml). PCR conditions were 2 minutes at $50^{\circ} \mathrm{C}, 10$ minutes at $95^{\circ} \mathrm{C}$, followed by 35 cycles of 15 seconds at $95^{\circ} \mathrm{C}$ and $60^{\circ} \mathrm{C}$ for 1 minute using an $\mathrm{ABI}$ PRISM 7900 HT (Applied Biosystems). PCR amplification of the house-keeping gene encoding glyceraldehyde-3phosphate dehydrogenase (Mm999999915_gl) was performed during each run for each sample to allow normal- ization between samples. A nontemplate control was included in all of the experiments to evaluate DNA contamination of isolated RNA and reagents. The results were analyzed by comparative threshold cycle method. The relative expression level of each sample was expressed as fold change from normal control.

\section{Preparation of Single-Cell Suspension from Cornea}

Seventy-two hours after suture placement, neovascularized corneas were pooled (10 corneas/group), teased with scissors, and digested by incubation with collagenase D (Sigma-Aldrich, St. Louis, MO) at $37^{\circ} \mathrm{C}$ for 1 hour. Total cells were collected after passing through a steel mesh.

\section{Flow Cytometry}

On blockade by anti-FcR monoclonal antibody, singlecell suspensions of neovascularized corneas were labeled with fluorescein isothiocyanate (FITC)-conjugated rat anti-mouse Gr-1 and PE-conjugated rat anti-mouse CD11b (granulocyte/monocyte/macrophage marker), and VECs were labeled with PE-conjugated rat antimouse PD-L1 or FITC-conjugated rat anti-mouse CD80 or FITC-conjugated rat anti-mouse VEGFR2. Cells labeled with isotype antibodies (FITC-conjugated rat immunoglobulin G2b, PE-conjugated rat immunoglobulin G2b, PE-conjugated rat immunoglobulin G2a, and FITC-conjugated rat immunoglobulin G2) were used as controls. After staining at $4^{\circ} \mathrm{C}$ for 30 minutes, the cells were analyzed by flow cytometry (Beckman Coulter, Brea, CA). All antibodies were purchased from BD Pharmingen (San Diego, CA) or eBioscience (San Diego, CA).

\section{Culture of Primary Mouse VECs and MS1 Cell Line}

Mouse heart and lung endothelial cells were isolated from homogenates of heart and lung tissue using sequential selection with anti-CD31- and anti-CD102-coated magnetic beads as described previously. ${ }^{22}$ Briefly, tissue from 9- to 15-day-old mice was digested with type-1 collagenase (Worthington Biochemical, Lakewood, NJ), mechanically dissociated, passed through a 70- $\mu \mathrm{m}$ filter, spun, resuspended, and incubated with sheep anti-rat Dynal beads (Invitrogen) that were coated with rat antimouse CD31 (clone MEC13.3; BD Biosciences, San Diego, CA). Cells were magnetically selected and plated on fibronectin-coated (Invitrogen) tissue culture dishes in RPMI complete media supplemented with $100 \mu \mathrm{g} / \mathrm{mL}$ endothelial mitogen (Endothelial cell growth supplement; Biomedical Technologies, Stoughton, MA). After 5 to 7 days, endothelial monolayers were trypsinized and further selected with sheep anti-rat Dynal beads coated with anti-CD102 (MIC2/4; BD Biosciences). Endothelial cells were greater than $97 \%$ pure based on flow cytometry analysis of co-expression of CD105 and CD102, and were used within two passages.

The MS1 cell line is a pancreatic islet endothelial cell line from a C57BL/6 mouse (CRL-2279; ATCC, Manas- 
sas, VA). MS1 cells were maintained in monolayer culture in Dulbecco's modified Eagle's medium supplemented with $1.0 \mathrm{~g} / \mathrm{L} \mathrm{D}$-glucose, $862 \mathrm{mg} / \mathrm{L}$ L-glutamine, $110 \mathrm{mg} / \mathrm{L}$ sodium pyruvate, and $10 \%$ heat-inactivated bovine serum at $37^{\circ} \mathrm{C}$ in $5 \% \mathrm{CO}_{2}$-containing humidified air.

\section{Small-Interfering RNA Transfection and Cell Proliferation Assay}

MS1 cells $\left(2 \times 10^{5}\right.$ cells per well) were plated in a 6-well plate and incubated in $2 \mathrm{~mL}$ antibiotic-free growth medium overnight to reach $60 \%$ confluency. A total of $6 \mu \mathrm{L}$ small-interfering RNA (siRNA) duplex and $6 \mu \mathrm{L}$ transfection reagent were diluted in $100 \mu \mathrm{L}$ siRNA transfection medium following the manufacturer's protocol (Santa Cruz Biotechnology, Santa Cruz, CA). The diluted siRNA and transfection reagent were mixed and incubated at room temperature for 45 minutes. The cells were washed with transfection medium, and then the diluted siRNA mixture was added to the 6 -well plates for 6 hours. Then $1 \mathrm{~mL}$ of normal growth medium containing 2 times the normal serum and antibiotic concentration was added without removing the transfection mixture and the cells were incubated for an additional 24 hours. Cells then were harvested and suspended in normal growth medium. For cell proliferation analysis, cells were placed in a 96-well plate (5000 cells/well) with normal growth medium. After another 24 to 48 hours, bromodeoxyuridine was added and cell proliferation was measured using the bromodeoxyuridine incorporation assay according to the manufacturer (Millipore, Billerica, MA). To measure VEGFR2 expression levels, cells were placed in a 6 -well plate $\left(1-2 \times 10^{5} /\right.$ well). Cells were harvested at 6 hours for reverse transcription-polymerase chain reaction and at 48 hours for flow cytometry.
Corneal Epithelial Cell Co-Cultured with VEC in a Non-Contact Manner

Corneal epithelial cells isolated from wild-type (WT) mice and PD-L $1^{-1-}$ knockout $(\mathrm{KO})$ mice were cultured in apical compartments of the Transwells (Corning Incorporated, Lowell, MA) with MS1 cells grown in the basal compartment of a 24-well plate. Five mice were used to generate corneal epithelial cells and cells were pooled in each group. All samples in each group were analysed in triplicate. First, MS1 cells were seeded $\left(5 \times 10^{3}\right.$ cells/well) in the absence of epithelial cells for 24 hours. When MS1 cells reached $30 \%$ confluence, the Transwells containing corneal epithelial cells $\left(0.6,1.25\right.$ or $2.5 \times 10^{5} /$ well) were added to 24 -well plates. Cells subsequently were co-cultured for an additional 72 hours, and the number of living cells in the different groups were counted by using a hematocytometer with trypan blue in a blinded fashion at that time.

\section{Immunohistochemical Studies}

Full-thickness corneal tissues were fixed in acetone for 15 minutes at room temperature. After Fc blocking (CD16/ CD31), FITC-conjugated rat anti-mouse CD31 (Santa Cruz Biotechnology, Santa Cruz, CA) was applied at $4^{\circ} \mathrm{C}$ overnight. After washing in phosphate-buffered saline for 5 to 10 minutes, the samples were covered with mounting medium (Vector Laboratories, Burlingame, CA).

To quantify corneal angiogenesis, digital pictures of corneal flat-mounts were taken using an image analysis system (Spot Image Analysis; Diagnostic Instruments, Sterling Heights, MI). The areas covered by CD31 ${ }^{\text {high }}$ (blood vessels) were measured morphometrically using National Institutes of Health ImageJ software (version 1.34s, http://rsb.info.nih.gov/ij, last ac-
A

PD-L1
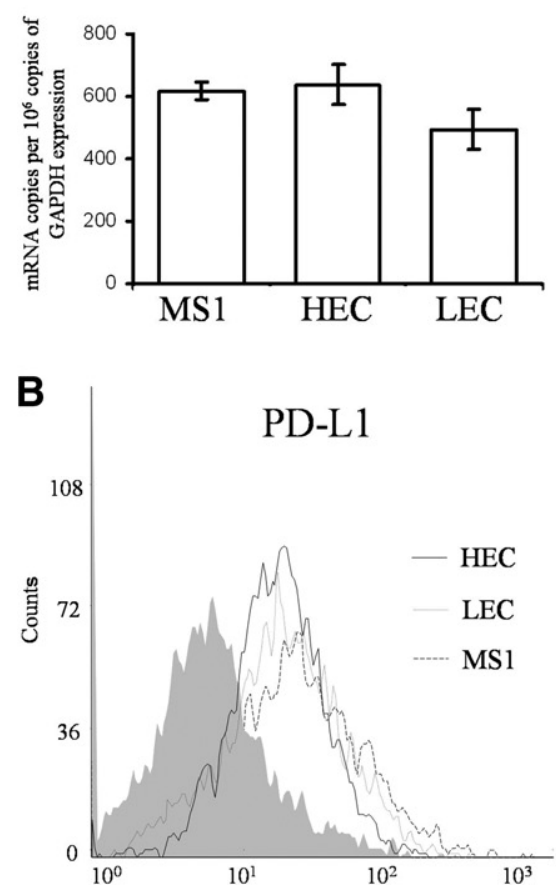

CD80
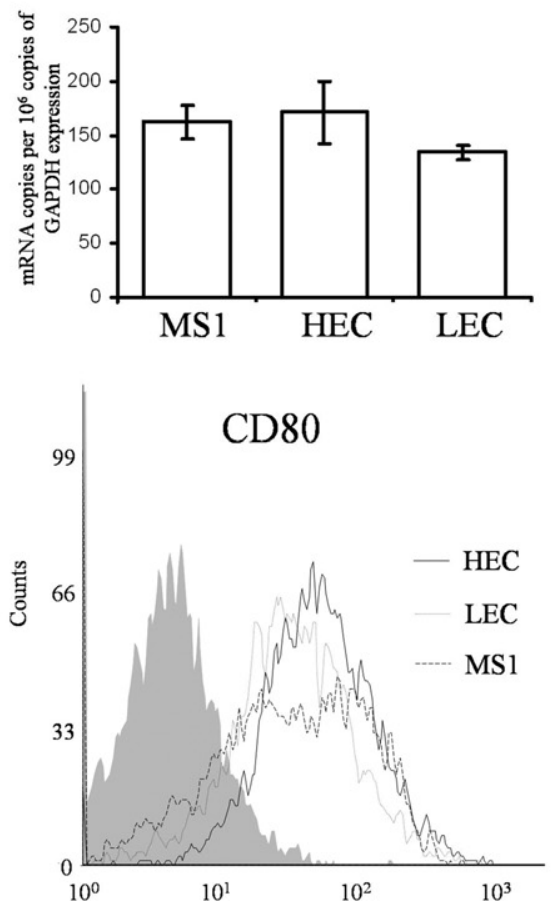

PD1

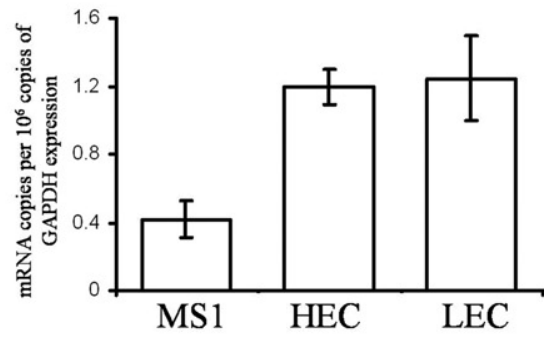

Figure 1. VECs express PD-L1 and CD80, but not PD-1. A: We isolated mRNA from MS1 cells, and primary VECs from heart (HEC) and lung (LEC) in vitro. The mRNA expression levels of PD-L1, CD80, and PD-1 in these three types of VECs were tested by real-time reverse transcriptionpolymerase chain reaction and represented as mRNA copies per $10^{6}$ copies of glyceraldehyde-3-phosphate dehydrogenase (GAPDH) expression. B: The protein expression levels of PD-L1 and CD80 in these cells were detected by flow cytometry after staining with anti-PD-L1-FITC or anti-CD80-FITC. Isotype control is shown as a gray area. 
cessed June 08, 2006). The total corneal area was outlined using the innermost vessel of the limbal arcade as the border. The vessel density was calculated by the proportion of neovascularized area to the whole corneal area.

\section{Statistics}

All data are expressed as means \pm standard error of the mean. Statistical significance between the experimental groups and the control group was analyzed by the twotailed $t$-test with Prism (version 4.0; GraphPad, San Diego, CA)

\section{Results}

\section{Expression of PD-L1 and Its Receptors on VECs}

We first studied the expression of PD-L1 and its receptors, CD80 and PD-1, on murine primary VECs from lung and heart, as well as the MS1 cell line. As shown in Figure 1A, all these VECs expressed similar levels of either PD-L1 or CD80 mRNA. Consistently, we also found that all these VECs had similar levels of PD-L1 or CD80 protein expression by flow cytometry (Figure 1B). The mRNA expression level of PD-1 was extremely low and the protein expression of PD-1 could not be detected in any of these three cell types (data not shown). We found that the expression levels of PD-L1 and CD80 were comparable among the murine VECs from different tissues, including primary VECs from the lung and heart, and MS1 cells; therefore, to maintain homogeneous conditions, we chose MS1 cells in the following in vitro functional assays.

\section{Inhibition of PD-L1 and CD80 Expression Up-Regulates VEGFR2 Expression Level and VEC Proliferation}

We transfected PD-L1 siRNA or CD80 siRNA into MS1 cells to inhibit PD-L1 or CD80 expression, respectively. As shown in Figure 2A, the mRNA expression levels of PD-L1 or CD80 were suppressed by approximately $75 \%$ at 48 hours after transfection, compared to cells transfected with control siRNA. Data from both real-time PCR and flow cytometry showed that MS1 cells transfected with PD-L1 or CD80 siRNA were expressing a significantly higher level of VEGFR2 compared to MS1 cells transfected with control siRNA (Figure 2, B and C). However, there was no significant difference in VEGFA expression among the groups (Figure $2 \mathrm{~B}$ ). Furthermore, the proliferation of MS1 cells was increased significantly after transfection with PD-L1 siRNA [optical density (OD) value, $0.85 \pm 0.02]$ or CD80 siRNA (OD value, $0.83 \pm$ 0.02 ), compared to the control siRNA group (OD value,
A

PD-L1

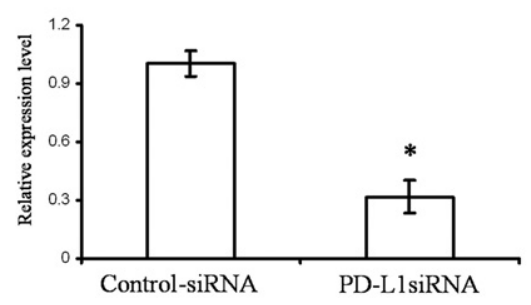

B

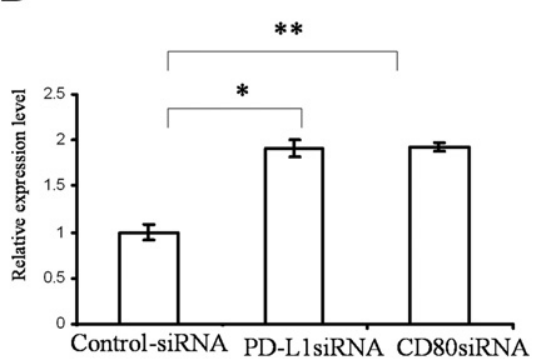

C

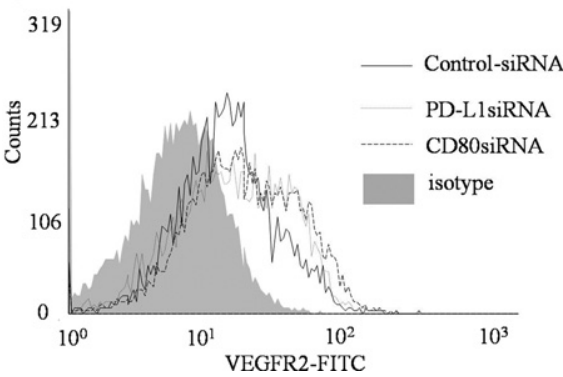

CD80

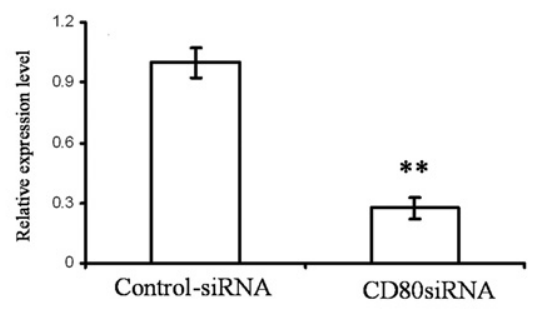

VEGFA

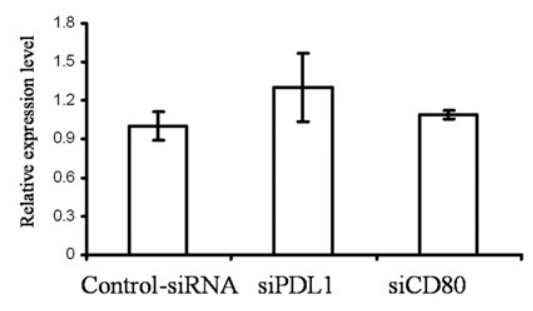

D

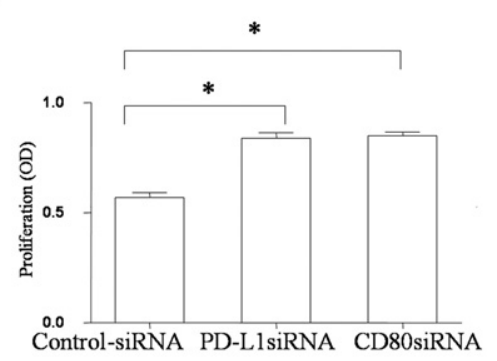

Figure 2. Inhibition or PD-L1 and CD80 expression up-regulates VEGFR2 expression and VEC proliferation. PD-L1 siRNA, CD80 siRNA, or control siRNA were transfected into MS1 cells for 48 hours. A: Total mRNA were isolated from different groups. The relative mRNA expression levels of PD-L1 and CD80 were tested for the efficacy of the transfections. B: After further incubation in the normal culture medium for 6 hours, the mRNA expression levels of VEGFR2 and VEGFA in MS1 from the different groups were analyzed. Data were normalized to glyceraldehyde-3 phosphate dehydrogenase mRNA and values were expressed as the fold change over the control siRNA-transfected group. C: After 24 hours incubation, the protein expression levels of VEGFR2 in different groups were measured by flow cytometry after staining with FITC-conjugated VEGFR2. Isotype control is shown as a gray area. D: The level of cell proliferation was measured using the bromodeoxyuridine incorporation assay after 36 hours of further incubation after transfections. OD, optical density. The results are depicted as the mean ( \pm standard error of the mean) of 4 samples per group. Data are representative of three independent experiments $\left({ }^{*} P<0.05,{ }^{* * *} P<0.01, t\right.$-test $)$. 
A
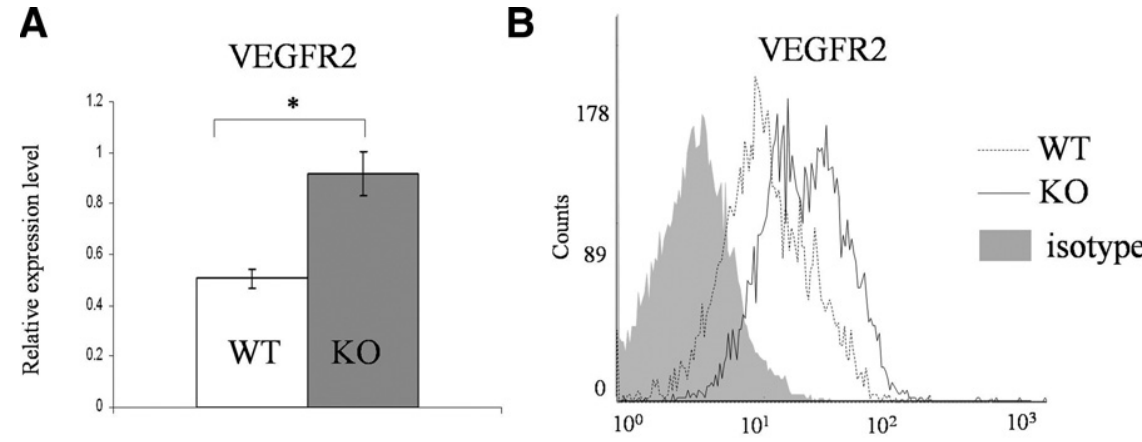

C

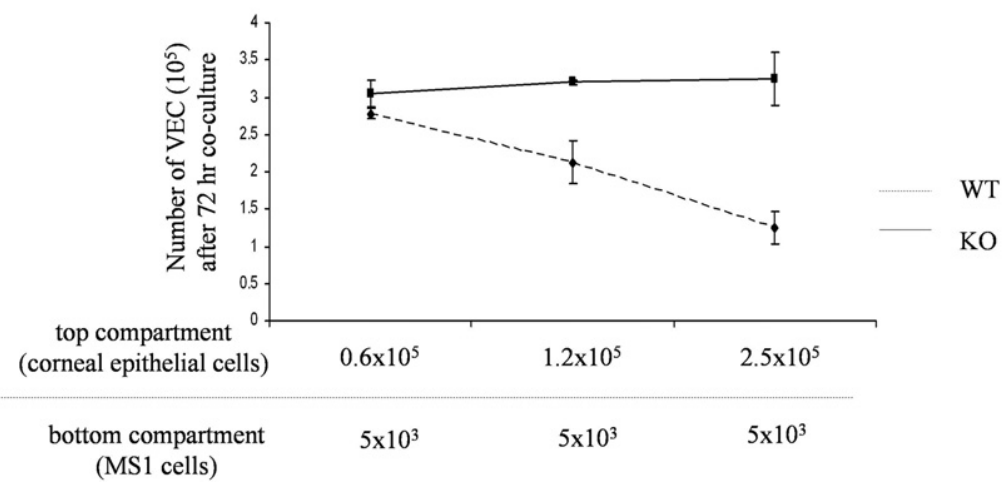

Figure 3. The expression of PD-L1 by corneal epithelial cells inhibits VEC proliferation in a dose-dependent and contact-independent man ner. Corneal epithelial cells isolated from WT and PD-L1 ${ }^{-/-}$mice $\left(1.25 \times 10^{5} /\right.$ well $)$ were cultured in apical compartments of Transwells with MS1 cells $\left(5 \times 10^{3}\right.$ cells/well $)$ seeded in the basal compartment of a 24 -well plate 24 hours prior. After 48 hours co-culture, the MS1 cells were harvested. A: The mRNA expression levels of VEGFR2 in MS1 from the different groups were analyzed. Data were normalized to glyceraldehyde-3-phosphate dehydrogenase mRNA and values were expressed as the fold change between the WT and PD-L1 ${ }^{-/-}$groups. The results are depicted as the mean ( \pm standard error of the mean) of 3 samples per group $\left({ }^{*} P<0.05, t\right.$-test $)$. B: The protein expression levels of VEGFR2 in different groups were measured by flow cytom etry after staining with FITC-conjugated VEGFR2. Isotype control is shown as a gray area. C: We loaded different amount of corneal epithelial cells in the apical compartments $(0.6 \times$ $10^{5}, 1.25 \times 10^{5}$, or $\left.2.5 \times 10^{5}\right)$ and co-cultured with the MS1 cells $\left(5 \times 10^{3}\right.$ cells/well $)$ seeded in the basal compartment for 72 hours. The numbers of live cells using trypan blue in the different groups were counted by a hematocytometer in a blinded fashion. The results are depicted as the mean ( \pm standard error of the mean) of 3 samples per group. Data are representative of two independent experiments.
$0.57 \pm 0.02$ ) (Figure $2 \mathrm{D}$ ), suggesting that the expression of PD-L1 and CD80 inhibits the proliferation of VECs, which may be associated with the down-regulation of VEGFR2 expression.

\section{PD-L1 Expression on Corneal Epithelial Cells Inhibits VEC Proliferation in a Dose-Dependent Contact-Independent Manner}

Because corneal epithelial cells have a high expression of PD-L1, we investigated whether the PD-L1 expression in corneal epithelial cells affects the proliferation of VECs in corneal stroma. Because there is a basement membrane that separates the corneal epithelial layer and the corneal stroma anatomically, we co-cultured VECs with corneal epithelial cells in a Transwell system to mimic in vivo conditions. Our data from real-time PCR and flow cytometry show that MS1 cells co-cultured with PD-L1 $1^{+/+}$WT corneal epithelial cells had a significant lower expression level of VEGFR2 as compared to the MS1 cells co-cultured with $\mathrm{PD}-\mathrm{L}^{-1-}$ corneal epithelial cells (Figure 3, A and B). Consistently, the MS1 cells co-cultured with PD-L $1^{+/+}$WT corneal epithelial cells had a significantly lower proliferation rate, as compared to the MS1 cells co-cultured with PD$\mathrm{L}^{-1-}$ corneal epithelial cells. This inhibitory effect was enhanced as the number of PD-L $1^{+/+}$epithelial cells was increased (Figure 3C), these results suggest that the PD-L1 expression by corneal epithelial cells inhibits the proliferation of VECs in a dose-dependent and contact-independent manner.

\section{Promotion of Corneal Angiogenesis in $\mathrm{PD}-\mathrm{L}^{-/-}$ KO Mice after Suture Placement}

To confirm the inhibitory effect of PD-L1 on angiogenesis in vivo, we evaluated corneal NV for 2 weeks after suture placement in corneas of WT versus PD-L1 $1^{-1-} \mathrm{KO}$ mice. We found that there was a significantly higher level of angiogenic response in the PD-L1 $1^{-1-} \mathrm{KO}$ group, compared to the WT group (Figure 4, A and B). Accordingly, the density of $\mathrm{CD} 31^{+}$blood vessels was enhanced significantly in the PD-L $1^{-1-}$ group relative to the WT group (22.1\% $\pm 2 \%$ vs $11.3 \% \pm 1 \% ; n=6$ per group; $P<0.01$; Figure 4, C and D).

We further compared mRNA expression levels of VEGFR2, the principal pro-angiogeneic VEGF-R, in PD$\mathrm{L}^{-1-}$ and WT corneas at 24 hours and 72 hours after suture placement. As seen in Figure 4E, the mRNA expression levels of VEGFR2 was significantly increased more than twofold in the $\mathrm{PD}-\mathrm{L} 1^{-1-}$ group compared to the WT corneas.

Finally, we compared the levels of inflammatory cytokines and the infiltration of innate immune cells between $\mathrm{PD}-\mathrm{L} 1^{-1-}$ and WT mice to determine whether the observed differences in the angiogeneic response were secondary to PD-L1-mediated regulation of the inflammatory response. As shown in Figure 5, there were no significant differences in the mRNA expression levels of $\mathrm{IL}-1 \alpha$, IL- $1 \beta$, or TNF- $\alpha$ between the two groups at either 24 hours or 72 hours. Moreover, there was no significant difference in the percentage of infiltrating Gr- $1^{+} \mathrm{CD} 11 \mathrm{~b}^{+}$ neutrophils $(22.01 \%$ vs $20.30 \%)$ or Gr- $1^{-} \mathrm{CD}^{-11 b^{+}}$macrophages $(8.84 \%$ vs $9.43 \%$ ) in the corneas at 72 hours between $\mathrm{PD}-\mathrm{L}^{-1-}$ and WT groups, suggesting that 
A

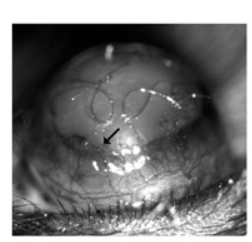

WT
B

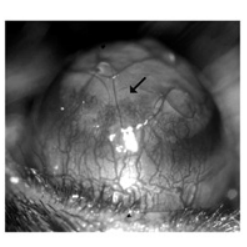

$\mathrm{KO}$

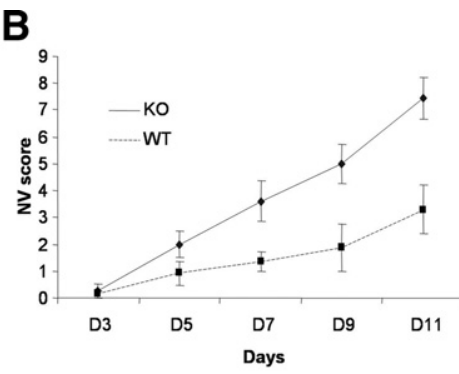

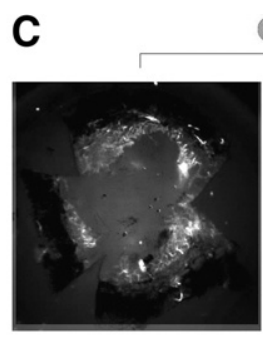

WT
CD31

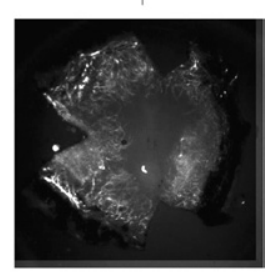

$\mathrm{KO}$
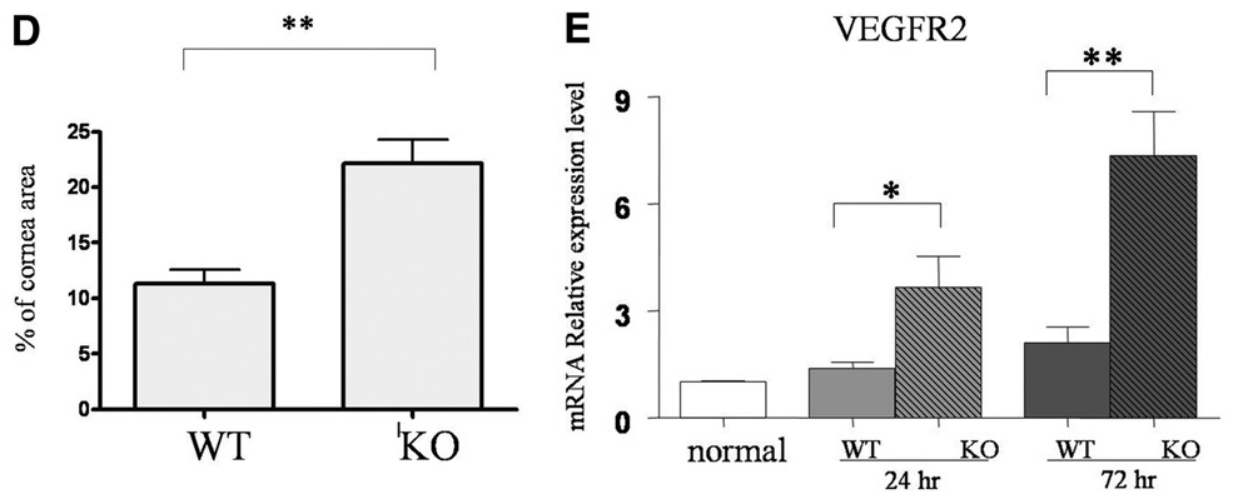

Figure 4. Promotion of corneal angiogenesis and VEGFR2 expression in PD-L1 ${ }^{-/}$mice after suture placement. A: Corneal NV was induced in WT and PD-L1 ${ }^{-/-}$ mice (arrows show the upper margin of the NV area). B: In a masked fashion, corneal NV was scored biomicroscopically with a slit-lamp using a grid system. Values are expressed as the mean ( \pm standard error of the mean) of 6 corneas. C: On day 14, whole corneas were dissected and stained with anti-CD31-FITC (shown in gray scale). D: The density of blood vessels $\left(\mathrm{CD} 31^{+}\right)$covering the cornea was analyzed. Values are expressed as the mean $( \pm$standard error of the mean) of 6 corneas per treatment group $\left({ }^{* *} P<0.01\right.$ vs vehicle-treated group). E: The mRNA expression level of VEGFR2 on the corneas was determined 24 hours or 72 hours after suture placement. Data were normalized to glyceraldehyde-3-phosphate dehydrogenase mRNA and values were expressed as the fold change over normal control corneas. Results represent the mean ( \pm standard error of the mean) of 4 samples per group (one cornea per sample, ${ }^{*} P<0.05$, ${ }^{* *} P<0.01$, $t$-test). Data are representative of two independent experiments.

PD-L1 inhibition of angiogenesis was not primarily caused by regulation of the inflammatory response.

\section{Discussion}

NV of the avascular cornea is a significant complication associated with many forms of corneal pathology. Maintain- ing this avascularity by active exclusion of blood vessels is an important aspect of maintaining corneal clarity and has been termed corneal angiogenesis privilege. Here, we demonstrate that PD-L1 expression by corneal epithelial cells and VECs plays an important role in the regulation of VEC proliferation, which in turn controls corneal angiogenesis.

\section{A}

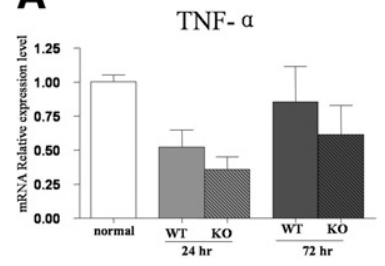

B

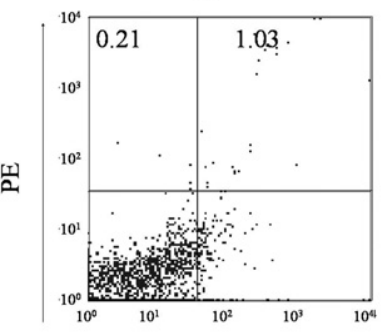

Fitc
IL-1 a

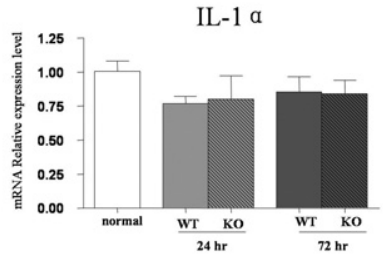

$\mathrm{PD}-\mathrm{L1}^{-/-\mathrm{KO}}$

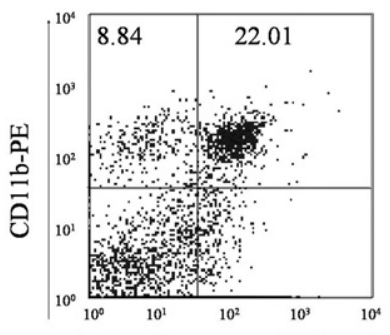

IL-1 $\beta$

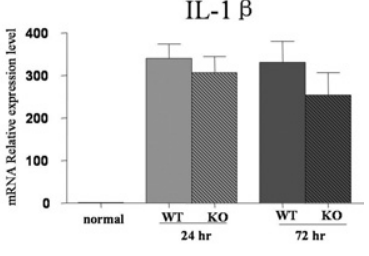

WT

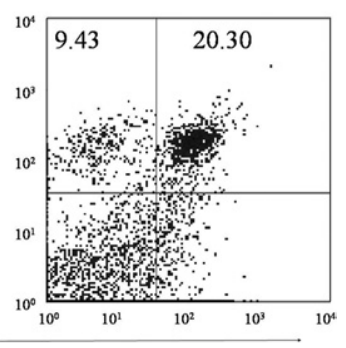

Gr-1-Fitc

Figure 5. PD-L1 regulation of corneal angiogenesis is not primarily owing to regulation of inflammatory cytokine expression. Intrastromal sutures were placed into the corneas of WT mice and PD-L1 ${ }^{-/}$mice to induce angiogenesis. A: Whole corneas were harvested at 24 hours or 72 hours, followed by RNA isolation. The mRNA expression levels of TNF- $\alpha$, IL- $1 \alpha$, and IL- $1 \beta$ were determined by real-time reverse transcription-polymerase chain reaction. Data were normalized to glyceraldehyde-3-phosphate dehydrogenase mRNA and values were expressed as the fold change over normal control corneas. Results represent the mean ( \pm standard error of the mean) of 4 samples per group (one cornea per sample, $t$-test). Data are representative of two independent experiments. B: Corneas were harvested from WT and PD-L1 ${ }^{-/-}$mice at 72 hours after suture placement and digested by using collagenase D. Similar amounts of cells were harvested from corneas in each group. Cells were double-stained with anti-CD11b-PE and anti-Gr-1-FITC antibodies, and analyzed by flow cytometry. Gr- $1^{+} \mathrm{CD} 11 \mathrm{~b}^{+}$neutrophil and Gr- $1^{-} \mathrm{CD} 11 \mathrm{~b}^{+}$macrophage populations were compared between WT and PD-L1 ${ }^{-/}$groups. 
The expression of PD-L1 on VECs has been related to the negative regulation of $\mathrm{T}$ cells. ${ }^{17,18}$ In this study, we demonstrate that VECs not only express PD-L1, but also B7-1 (CD80), one of its receptors. The interaction between PD-1 and PD-L1 is well established as playing a vital role in controlling T-cell responses in a variety of contexts, from development to autoimmunity to microbial pathogenesis to tumor immunity. ${ }^{9}$ However, the functional significance of the interaction between PD-L1 and CD80 is only beginning to be understood. Here, we demonstrate that the inhibition of either PD-L1 or CD80 expression in VECs, with siRNA transfection, similarly increases VEGFR2 expression and cell proliferation. This is notable because the expression of VEGFR2 is known to play a pivotal role in endothelial cell proliferation and differentiation. ${ }^{23}$ These results suggest that the expression of PD-L1 and CD80 in VECs may have negative regulatory effects on angiogenesis. Our studies did not investigate the mechanisms by which PD-L1 and CD80 regulate VEGFR2 expression; however, recent studies have shown that CD80-mediated signaling pathways inhibit extracellular signal-regulated kinases activation, ${ }^{24}$ which is important for VEGFR2 expression through activation of the E26 transformation-specific-1 transcription factor. ${ }^{25}$ Extracellular signal-regulated kinases activation is also a critical step for phosphorylated VEGFR2 to enhance VEC proliferation. ${ }^{26}$ Furthermore, because both PD-L1 and CD80 are expressed by VECs, we speculate that PD-L1 ligation may occur both in an autocrine and paracrine manner.

The intact corneal epithelium can suppress corneal hemangiogenesis. ${ }^{27}$ Our laboratory has demonstrated that PD-L1, but not CD80 and PD-1, is expressed constitutively at very high levels on normal corneal epithelial cells. 8,28 Therefore, we hypothesized that the expression of PD-L1 by corneal epithelial cells regulates corneal NV, and that this inhibitory effect is related to the ligation of CD80 on VECs. Accordingly, we tested the proliferation of VECs when they were co-cultured with corneal epithelial cells in a Transwell system, we found decreased VEGFR2 expression levels and impaired cell proliferation of VECs when the VECs were co-cultured with corneal epithelia cells. This suppression effect was in a dosedependent and contact-independent manner, and was attenuated when the corneal epithelial cells were derived from PD-L1-deficient mice. How could PD-L1 exert its effects in a contact-independent fashion? Several studies have detected a soluble form of PD-L1 in sera from patients. It is possible that corneal epithelial cells could secrete a soluble form of PD-L1, which may mediate these effects. ${ }^{29}$

We further evaluated the anti-angiogenic effect of PD-L1 in vivo. Consistently, we observed an increased expression level of VEGFR2 and enhanced corneal angiogenesis in the PD-L1 $1^{-1-}$ mice versus WT mice after suture placement. Our previous studies have shown that infiltrating inflammatory innate immune cells contribute to corneal angiogenesis. ${ }^{20,30}$ These infiltrating inflammatory cells, mainly neutrophils and macrophages, also present costimulatory molecules (eg, CD80) to induce or amplify immune responses. However, we did not find a signifi- cant increase in expression of inflammatory cytokines (IL-1 $\beta, \mathrm{IL}-1 \alpha$, and TNF- $\alpha$ ) or infiltrating cells in PD-L $1^{-/-}$ $\mathrm{KO}$ versus WT mice after induction of inflammation. Although these data cannot rule out subtle differences in the inflammatory response between PD- $\mathrm{L} 1^{-1-}$ and WT mice, we believe they do suggest that PD-L1 regulation of angiogenesis is not principally through suppression of inflammation, but rather an interaction between VECs and corneal epithelial PD-L1 with CD80 in an autocrine or paracrine manner.

Although our data demonstrate that deletion of PD-L1 results in an amplified angiogenic response in the inflamed cornea, we have not observed corneal vascularization in PD-L1 KO mice in a static state. This suggests that PD-L1 sets a higher threshold for angiogenic responses under robust angiogenic stimulation, but not in the normal uninflamed state.

In conclusion, this study provides novel data expanding the immunoregulatory function of PD-L1 to the regulation of angiogenesis, thereby suggesting a novel therapeutic indication for PD-L1 modulation.

\section{References}

1. Cursiefen C, Chen L, Saint-Geniez M, Hamrah P, Jin Y, Rashid S, Pytowski B, Persaud K, Wu Y, Streilein JW, Dana R: Nonvascular VEGF receptor 3 expression by corneal epithelium maintains avascularity and vision. Proc Natl Acad Sci U S A 2006, 103:11405-11410

2. Ambati BK, Nozaki M, Singh N, Takeda A, Jani PD, Suthar T, Albuquerque RJ, Richter E, Sakurai E, Newcomb MT, Kleinman ME, Caldwell RB, Lin Q, Ogura Y, Orecchia A, Samuelson DA, Agnew DW, St Leger J, Green WR, Mahasreshti PJ, Curiel DT, Kwan D, Marsh H, Ikeda S, Leiper LJ, Collinson JM, Bogdanovich S, Khurana TS, Shibuya M, Baldwin ME, Ferrara N, Gerber HP, De Falco S, Witta J, Baffi JZ, Raisler BJ, Ambati J: Corneal avascularity is due to soluble VEGF receptor-1. Nature 2006, 443:993-997

3. Streilein JW, Yamada J, Dana MR, Ksander BR: Anterior chamberassociated immune deviation, ocular immune privilege, and orthotopic corneal allografts. Transplant Proc 1999, 31:1472-1475

4. Niederkorn JY: The immune privilege of corneal allografts. Transplantation 1999, 67:1503-1508

5. Cursiefen C, Masli S, Ng TF, Dana MR, Bornstein P, Lawler J, Streilein JW: Roles of thrombospondin-1 and -2 in regulating corneal and iris angiogenesis. Invest Ophthalmol Vis Sci 2004, 45:1117-1124

6. Hayashi T, Yamagami S, Tanaka K, Yokoo S, Usui T, Amano S, Mizuki $\mathrm{N}$ : Immunologic mechanisms of corneal allografts reconstituted from cultured allogeneic endothelial cells in an immune-privileged site. Invest Ophthalmol Vis Sci 2009, 50:3151-3158

7. Hori J, Wang M, Miyashita M, Tanemoto K, Takahashi H, Takemori T, Okumura K, Yagita H, Azuma M: B7-H1-induced apoptosis as a mechanism of immune privilege of corneal allografts. J Immunol 2006, 177:5928-5935

8. Shen L, Jin Y, Freeman GJ, Sharpe AH, Dana MR: The function of donor versus recipient programmed death-ligand 1 in corneal allograft survival. J Immunol 2007, 179:3672-3679

9. Keir ME, Butte MJ, Freeman GJ, Sharpe AH: PD-1 and its ligands in tolerance and immunity. Annu Rev Immunol 2008, 26:677-704

10. Greenwald RJ, Freeman GJ, Sharpe AH: The B7 family revisited. Annu Rev Immunol 2005, 23:515-548

11. Dong H, Zhu G, Tamada K, Chen L: B7-H1, a third member of the B7 family, co-stimulates T-cell proliferation and interleukin-10 secretion. Nat Med 1999, 5:1365-1369

12. Yamazaki T, Akiba H, Iwai H, Matsuda $H$, Aoki M, Tanno $Y$, Shin $T$, Tsuchiya H, Pardoll DM, Okumura K, Azuma M, Yagita H: Expression of programmed death 1 ligands by murine T cells and APC. J Immunol 2002, 169:5538-5545

13. Loke P, Allison JP: PD-L1 and PD-L2 are differentially regulated by Th1 and Th2 cells. Proc Natl Acad Sci U S A 2003, 100:5336-5341 
14. Nakazawa A, Dotan I, Brimnes J, Allez M, Shao L, Tsushima F, Azuma M, Mayer L: The expression and function of costimulatory molecules $\mathrm{B} 7 \mathrm{H}$ and $\mathrm{B} 7-\mathrm{H} 1$ on colonic epithelial cells. Gastroenterology 2004 , 126:1347-1357

15. Stuart PM, Pan F, Plambeck S, Ferguson TA: FasL-Fas interactions regulate neovascularization in the cornea. Invest Ophthalmol Vis Sci 2003, 44:93-98

16. Reed MJ, Iruela-Arispe L, O'Brien ER, Truong T, LaBell T, Bornstein P, Sage EH: Expression of thrombospondins by endothelial cells. Injury is correlated with TSP-1. Am J Pathol 1995, 147:1068-1080

17. Eppihimer MJ, Gunn J, Freeman GJ, Greenfield EA, Chernova T, Erickson J, Leonard JP: Expression and regulation of the PD-L1 immunoinhibitory molecule on microvascular endothelial cells. Microcirculation 2002, 9:133-145

18. Mazanet MM, Hughes CC: $\mathrm{B} 7-\mathrm{H} 1$ is expressed by human endothelial cells and suppresses T cell cytokine synthesis. J Immunol 2002 , 169:3581-3588

19. Latchman YE, Liang SC, Wu Y, Chernova T, Sobel RA, Klemm M, Kuchroo VK, Freeman GJ, Sharpe AH: PD-L1-deficient mice show that PD-L1 on T cells, antigen-presenting cells, and host tissues negatively regulates T cells. Proc Natl Acad Sci U S A 2004, 101:10691-10696

20. Dana MR, Zhu SN, Yamada J: Topical modulation of interleukin-1 activity in corneal neovascularization. Cornea 1998, 17:403-409

21. Jin Y, Shen L, Chong EM, Hamrah P, Zhang Q, Chen L, Dana MR: The chemokine receptor CCR7 mediates corneal antigen-presenting cell trafficking. Mol Vis 2007, 13:626-634

22. Rodig N, Ryan T, Allen JA, Pang H, Grabie N, Chernova T, Greenfield EA, Liang SC, Sharpe AH, Lichtman AH, Freeman GJ: Endothelial expression of PD-L1 and PD-L2 down-regulates CD8+ T cell activation and cytolysis. Eur J Immunol 2003, 33:3117-3126

23. Takahashi T, Yamaguchi S, Chida K, Shibuya M: A single autophosphorylation site on KDR/FIk-1 is essential for VEGF-A-dependent activation of PLC-gamma and DNA synthesis in vascular endothelial cells. EMBO J 2001, 20:2768-2778

24. Dowling D, Hamilton CM, O'Neill SM: A comparative analysis of cytokine responses, cell surface marker expression and MAPKs in DCs matured with LPS compared with a panel of TLR ligands. Cytokine 2008, 41:254-262

25. Oettgen P: Regulation of vascular inflammation and remodeling by ETS factors. Circ Res 2006, 99:1159-1166

26. Svensson S, Jirstrom K, Ryden L, Roos G, Emdin S, Ostrowski MC, Landberg G: ERK phosphorylation is linked to VEGFR2 expression and Ets-2 phosphorylation in breast cancer and is associated with tamoxifen treatment resistance and small tumours with good prognosis. Oncogene 2005, 24:4370-4379

27. Hori J, Streilein JW: Role of recipient epithelium in promoting surviva of orthotopic corneal allografts in mice. Invest Ophthalmol Vis Sci 2001, 42:720-726

28. Hamrah P, Huq SO, Liu Y, Zhang Q, Dana MR: Corneal immunity is mediated by heterogeneous population of antigen-presenting cells. $\mathrm{J}$ Leukoc Biol 2003, 74:172-178

29. Her M, Kim D, Oh M, Jeong H, Choi I: Increased expression of soluble inducible costimulator ligand (ICOSL) in patients with systemic lupus erythematosus. Lupus 2009, 18:501-507

30. Dana MR, Streilein JW: Loss and restoration of immune privilege in eyes with corneal neovascularization. Invest Ophthalmol Vis Sci 1996 37:2485-2494 